\title{
The development of a survey instrument to measure the barriers to the conduct and application of research in complementary and alternative medicine: a Delphi study
}

Yasamin Veziari ${ }^{1 *}$ (D, Saravana Kumar ${ }^{1}$ and Matthew Leach ${ }^{2}$

\begin{abstract}
Background: As Complementary and Alternative medicine (CAM) grows in popularity, there is overt recognition for research evidence to inform clinical practice. While various strategies have been trialed to overcome the barriers to such progress, it is important to first understand the extent to which these barriers impact the conduct and application of CAM research. This study aimed to garner consensus and agreement among CAM researchers and practitioners in refining and validating a preliminary survey instrument for measuring these barriers.

Methods: A three-round Delphi study was undertaken with 22 international experts of CAM research and practice. Round one gathered consensus on 30 statements listing barriers to the application and conduct of CAM research. Only rounds two and three sought consensus on revised statements. Any statement demonstrating $\geq 70 \%$ agreement on a four-point Likert scale was determined to have reached consensus.

Results: Consensus and agreement was achieved for all 30 statements listing the barriers to the application and conduct of research in CAM. Additional commentary by the Delphi participants directed whether a statement had to be split into two parts or reworded. This process resulted in the generation of the "BarrierS To the Application and Conduct of rEsearch" (OBSTACLES) instrument.

Conclusion: This study, the first of its kind on this topic, identified consensus and agreement on a series of evidenceinformed statements to measure the barriers to the conduct and application of research in CAM. The uniqueness of the oBSTACLES instrument is its ability to measure barriers across the evidence-based continuum.
\end{abstract}

Keywords: Complementary and alternative medicine, Evidence-based practice, Research, Barriers, Delphi study, Consensus

\section{Background}

Complementary and Alternative medicine (CAM) refers to a large group of healthcare practices that are not part of a country's own conventional medicine and are not integrated into mainstream healthcare of a country [1]. CAM therapies follow a holistic model of health with the practitioner-client relationship being an important element of practice [2]. This group of therapies employ interventions/techniques that promote the innate healing

\footnotetext{
* Correspondence: Yasamin.Veziari@mymail.unisa.edu.au

${ }^{1}$ School of Health Sciences, University of South Australia, North Terrace,

Adelaide, SA 5000, Australia

Full list of author information is available at the end of the article
}

ability of the body while retaining a core focus on individuality, holism, education and disease prevention [3]. The growing use of CAM across the globe [4] can be attributed to many factors, including but not limited to, the move towards holistic well-being, the recognition of the limitations associated with conventional medicine and the increasing discourse on the important contribution of CAM [5]. As the popularity of CAM has increased, so has the level of scrutiny about the evidence underpinning its effectiveness [6]; resulting in calls for increased research and an appraisal of the evidence [7, 8]. There is a view amongst the scientific community that CAM, unlike conventional medicine, is not underpinned by rigorous

(c) The Author(s). 2018 Open Access This article is distributed under the terms of the Creative Commons Attribution 4.0 International License (http://creativecommons.org/licenses/by/4.0/), which permits unrestricted use, distribution, and reproduction in any medium, provided you give appropriate credit to the original author(s) and the source, provide a link to the Creative Commons license, and indicate if changes were made. The Creative Commons Public Domain Dedication waiver (http://creativecommons.org/publicdomain/zero/1.0/) applies to the data made available in this article, unless otherwise stated. 
research [9], and this has resulted in calls for increased research and a balanced appraisal of the evidence in order to improve confidence in the CAM industry [7].

Parallel to the calls for more CAM research is the increasing recognition of the numerous barriers to conducting rigorous research in CAM [8]. Furthermore, translation of evidence into practice in CAM continues to face challenges [10-15] that result in persistent knowledge and practice gaps [16]. While the existence of these issues in CAM research and practice are recognised, there is no single instrument that adequately measures the barriers to the conduct of research and the application of research evidence in CAM. While some measures are available that explore the barriers to the utilisation of research in practice, such as the "Barriers to Research Utilization Scale" (BARRIERS) [17] and the "Evidence-Based practice Attitude and utilization SurvEy" (EBASE) [18], these tools do not address barriers pertinent to both the conduct of CAM research and the application of research evidence in CAM practice. The former is important, as the identification of barriers to the conduct of research is the necessary first step towards improving the quality of research in CAM [19]; identifying the barriers to the application of research is a necessary second step to ensuring that the findings of such research are utilised in clinical practice.

One factor possibly contributing to the slow uptake of evidence-based practice (EBP) in CAM may be that the barriers to the conduct of research and the application of research evidence in CAM are not well understood, or alternatively, have not been adequately measured. This highlights the need for an instrument that can identify the barriers to the conduct as well as the application of research in CAM. Identifying these obstacles to CAM research conduct / application will set the path to establishing a stronger research culture, building a quality evidence base, and improving the uptake of the best available evidence in CAM practice.

This research presents the third and final stage of a multi-method project designed to develop an instrument to measure the barriers to the conduct and application of research in CAM. Stage one of the project was a systematic review, the purpose of which was to synthesise the evidence pertaining to the barriers to the conduct and application of research in CAM [20]. The barriers that were unique to the conduct of research were captured within one of two categories: "capacity" and "culture". "Capacity" referred to barriers in the areas of access, competency and bias. Access related to funding, training, and skills; bias related to the inherent negative perceptions about CAM research; and competency referred to the skills, knowledge, experiences and research education of the CAM practitioner in terms of conducting research. Barriers identified within the "culture" category were broadly classified as values and complex systems. Values related to inherent practices, reluctance to engage with mainstream research, historical perspectives, and an educational model that places little emphasis on research evidence. Complex systems referred to the complexity underpinning CAM research, the inability to undertake blinding and/or use placebo controls, and the limited generalisability of findings.

The barriers to the application of research were similarly captured within the categories of "capacity" and "culture". Under "capacity" were barriers pertaining to access, competency and bias. Access related to limited resources, limited quality evidence and insufficient skills. Competency referred to the limited research skills of a practitioner and the inability to interpret and impart results. Within the sub-category of bias were negative perceptions of research, historical viewpoints leading to the antithesis of EBP in CAM, the lack of incentives in CAM research and lack of time. The category of "culture" captured a number of barriers relating to beliefs, lack of interest in research and infrequent use of bibliographic databases. All of these barriers to the conduct and application of research in CAM provided the necessary framework for a provisional survey instrument. Statements to be included in the provisional survey instrument were developed using a nominal group technique.

Stage two of the research project involved conducting a nominal group technique (NGT) [21]. The NGT brought together the findings from the systematic review, as well as the expertise of local CAM providers and researchers, to develop a preliminary list of statements for a pilot survey instrument. The experts were selected using purposive sampling; this ensured that participants with pertinent expertise and personal attributes were selected [22]. The nominal group technique consisted of 7 stages [21]: In stages 1 and 2 (welcome and deliberations), an A3 document containing a list of all barriers generated from the stage 1 systematic review were handed to the participants. Adjacent to these barriers were 72 examples of potential statements that reflected these barriers. This included 44 statements relating to the barriers to the conduct of research in CAM, and 28 statements relating to the barriers to the application of research in CAM. Each participant individually considered the issues to be deliberated. For stages 3 and 4 (generation of ideas/themes and discussions), each participant disclosed the results of their deliberations. The group discussed that it had understood the items that were put forward, and that all participants agreed that each of the statements were relevant barriers to the field of CAM. Attention was then directed towards the wording of the statements, of which some statements underwent minor editorial 
changes. In stages 5 and 6 (evaluation and consensus), ideas were evaluated; participants agreed upon ideas; and the list of 72 examples of potential statements presented in stage 2 evolved into 36 statements. For stage 7 (data refinement), the developed ideas were reworded and rearranged, and key constructs for themes that were suggested by the NGT participants were taken into consideration in the design of the preliminary survey instrument.

The outcome of the NGT was the development of a preliminary survey instrument consisting of 30 statements, with the conduct section of the instrument consisting of 16 statements, and the application section of the instrument comprising 14 statements. This instrument was referred to as "BarrierS To the Application and Conduct of rEsearch (oBSTACLES)". This preliminary instrument was later refined and validated through a Delphi study involving international experts in CAM. This manuscript reports the findings from this Delphi study.

\section{Aim}

The aim of this study was to refine and validate a preliminary survey instrument for measuring the barriers to the conduct and application of research in complementary and alternative medicine.

\section{Methods}

\section{Design}

\section{The Delphi study}

A three-round Delphi study was carried out for this research. This iterative approach provided a means of reaching consensus on a complex healthcare problem [23] in order to improve policy, research and clinical decision-making $[24,25]$. Accordingly, the study design was most suitable in addressing the aim of this study.

The Delphi method explores divergence in an iterative series of rounds in order to gain consensus from a panel of experts [25]. The rounds involve the presentation of an issue to a panel of experts to seek their opinion. Once all the participants respond, the data are summarised, and subject to the responses provided, a second round is sent out. First-round responses of participants are collated and analysed to form the second round and so on [26]. Repeat rounds are continued until consensus is reached [27]. Delphi studies do not have a set limit on the number of rounds to be undertaken. At the discretion of the researcher [28], two rounds [27] or more may be undertaken, with three rounds being common [29].

\section{Participants}

Establishing a panel of experts is a core requirement of a Delphi study [30]. For this research, the panel comprised of international experts in CAM research and practice, recruited from within three geographical areas where
CAM is widely practiced and researched, namely 1) Australasia, 2) Europe and 3) North America. A description of the participant inclusion and exclusion criteria is presented in Table 1.

\section{Sample size}

No guidance on appropriate sample sizes for Delphi studies [31] currently exists. Instead, the scope of the problem and available resources typically determines participant numbers [32, 33]. Furthermore, larger sample sizes do not necessarily equate with better responses, with data collection and analysis increasing in complexity with increasing sample size [27]. Given the specific focus of this research, the resources available and time constraints, a sample size of 21-24 was considered adequate [34]. Hence, this study aimed to recruit 7-8 participants per geographical region, which is similar to sample sizes reported in other Delphi studies $[35,36]$.

\section{Sampling and recruitment}

The sampling strategy encompassed three approaches: 1 . Stratification sampling to ensure adequate representation of each geographical region [37]; 2. Purposive sampling to enable the selection of participants based on their expertise and willingness to share their expertise [22], of which CAM experts were selected from countries where CAM is widely practiced and researched; and 3. Snowball sampling [38, 39], which identified additional CAM researchers and practitioners. The participants from stage 2 (NGT) were invited to suggest names of international or national researchers/practitioners who may have been able to contribute to this research. Additionally, where contact details could be obtained, authors of publications identified in stage 1 (systematic review) were invited to participate in this Delphi study.

Recruitment followed the Dillman Protocol [40]. An invitation email informed eligible participants about the proposed research and the importance of participating in all rounds to minimise attrition bias [41]. If there was no response to the invitation within 1 week, a second reminder was sent [42]; if there was still no response, it was assumed that the participant was unavailable, and no further attempts were made to contact the participant.

\section{Procedure}

\section{Data collection}

Once the desired number of participants had responded, participants were sent an invitation email containing an active hyperlink (this process was also followed for rounds two and three). Upon entering the survey, participants provided informed consent, and then proceeded to commence round one of the Delphi study. Participants were given 4 weeks to complete each round. 
Table 1 Overview of the participant inclusion \& exclusion criteria

\begin{tabular}{|c|c|c|}
\hline Inclusion criteria & & Exclusion criteria \\
\hline Researchers & Practitioners & Researchers/Practitioners \\
\hline $\begin{array}{l}\text { - Researchers who have a particularistic focus in CAM } \\
\text { research } \\
\text { - Researchers who preferably have a PhD } \\
\text { or a minimum of a post graduate qualification } \\
\text { - Researchers who have published in the area of expertise } \\
\text { - Researchers who have an appointment or affiliation with } \\
\text { a governing organisation }\end{array}$ & $\begin{array}{l}\text { - Practitioners from disciplines such as naturopathy, } \\
\text { homeopathy, herbalism, chiropractic, massage therapy or } \\
\text { acupuncture/TCM and osteopathy } \\
\text { - Practitioners working in CAM for at least } 10 \text { years } \\
\text { - Practitioners with a minimum of a post graduate } \\
\text { qualification } \\
\text { - Practitioners who have an appointment or affiliation } \\
\text { with a governing organization }\end{array}$ & $\begin{array}{l}\text { - Medical/health } \\
\text { researchers who research } \\
\text { CAM as an ancillary } \\
\text { - Practitioners of reiki, } \\
\text { meditation, aromatherapy } \\
\text { or hypnosis }\end{array}$ \\
\hline
\end{tabular}

Reminders were sent to late respondents (after 4 weeks), which included a 2-week extension to complete the round. Those that did not respond within the six-week period were classified as non-respondents, with no further attempts made to contact them. All three Delphi rounds used the online survey platform, SurveyMonkey ${ }^{\text {тм }}$ [43]. In the first round, participants were presented with all 30 statements (Table 2) from the preliminary survey instrument (i.e. derived from stage 2), with statements divided into two parts: barriers to the conduct of research (part A) and barriers to the application of research findings (part B). In rounds two and three, participants only received statements that had undergone some degree of revision (i.e. statements that had not yet been accepted or rejected by the majority of experts). All participants that participated in round one were given the opportunity to participate in rounds two and three.

\section{Data management and analyses}

For each round, participants were asked to review each statement and to provide a response to two questions. For the first question, The relevance of each item, participants were required to indicate the relevance of the proposed item on a 4-point Likert scale: category $0=$ not relevant, category 1 = relevant but requires major revision, category $2=$ relevant but requires minor revision, and category 3 = very relevant and requires no revision. For the second question, Any additional commentary, participants had to provide justification for their score, and if desired, provide additional comments. An example of these two questions is presented in Fig. 1.

Although the Delphi is a recognised technique for the investigation of opinions, it has no set agreement on consensus [23]. However, a clear definition of consensus prior to data analysis is important for credibility [33]. Therefore, as used in other health research [44-47],

Table 2 The Preliminary survey instrument

\section{Part A: Barriers to the conduct of research in CAM}

1. There are limited funding opportunities to conduct research in CAM

2. There are limited incentives for researchers to participate in CAM.

3. There are limited opportunities for CAM practitioners to contribute to CAM research.

4. There are limited numbers of dedicated CAM researchers

5. There are limited numbers of journal/grant reviewers with expert understanding of CAM.

6. There are limited large scale and long-term CAM research studies.

7. There are limited opportunities to recruit CAM research participants due to negative public attitudes towards CAM.

8. There are negative perceptions of CAM research amongst those outside the field of CAM.

9. There are limited opportunities to publish CAM research in scientific journals.

10. There is limited collaboration between CAM and other health researchers.

11. There are limited numbers of CAM researchers who have dedicated expertise in a CAM discipline.

12. There is limited recognition for the value of research within CAM.

13. There are limited opportunities for research skills development in CAM undergraduate education.

14. There are limited opportunities for CAM undergraduate students to contribute to CAM.

15. Traditional research designs are of limited value to test complex individualised CAM treatments.

16. The reductionist biomedical model of care is not compatible with the broader CAM holistic model of care.
Part B: Barriers to the application of research in CAM

17. CAM practitioners have limited access to research evidence.

18. CAM practitioners have limited access to research training.

19. CAM practitioners have limited awareness of clinical practice guidelines.

20. CAM practitioners have limited knowledge and skills to locate the best available research evidence.

21. Publication bias poses a challenge for CAM practitioners to locate evidence.

22. CAM practitioners have limited knowledge and skills to appraise research evidence.

23. CAM practitioners have limited knowledge and skills to apply research evidence to practice.

24. Inconsistencies within CAM research evidence are an obstacle to applying research evidence to practice.

25. CAM practitioners have limited knowledge and skills to communicate research findings to their patients.

26. CAM practitioners have limited time to apply research evidence to their practice.

27. CAM practitioners have limited financial incentives to use research evidence to inform their practice.

28. CAM practitioners have little professional obligation to use research evidence to inform their practice.

29. CAM practitioners have diverse views on what constitutes research evidence.

30. CAM practitioners are often faced with patient expectations that are contrary to research evidence. 
STATEMENT (e.g. There are limited funding opportunities to conduct research in CAM)

\begin{tabular}{|c|c|c|c|}
\hline Not relevant & $\frac{\text { Relevant but requires }}{\text { major revision }}$ & $\frac{\text { Relevant but requires }}{\text { minor revision }}$ & $\frac{\text { Very relevant and requires no }}{\text { revision }}$ \\
$\square$ & $\square$ & $\square$ & $\square$ \\
\hline
\end{tabular}

Fig. 1 The question and response format for each statement in each Delphi round

consensus for this study was defined as an agreement threshold $\geq 70 \%$. Where there was $\geq 70 \%$ agreement (as described below) but qualitative comments were provided, the research team reflected on these comments and made required changes to the statements.

Agreement was expressed as the percentage of agreement to the inclusion of each statement in the instrument. Where the sum of category 2 ("relevant but requires minor revision") and category 3 ("very relevant and requires no revision") was $\geq 70 \%$, the statement was included in the final instrument. If the sum of categories 2 and 3 was $<70 \%$, and the sum of category 1 ("relevant but requires major revision") and category 2 ("relevant but requires minor changes") was greater than the proportion of responses to category 0 ("not relevant"), the statement was amended (based on participant comments) and added to a consecutive Delphi round to allow further mediation by the panel. An agreement rating of $\geq 50 \%$ given to category 0 ("not relevant") meant the exclusion of the statement from the final instrument. Statements were also excluded if agreement had not been achieved within two rounds. This process was consistent with other Delphi studies [23, 48].

\section{Results}

\section{Participants}

The flow of participation from rounds one to three is presented in Fig. 2. One hundred and thirteen invitations were sent out. Round one had 26 responders, of which four incomplete surveys were excluded. Therefore, there was a total number of 22 participants with completed surveys in round one (20\% response rate). Rounds two and three had 10 participants each. Over the three rounds, the same group of experts were invited to participate in this Delphi study. There was some attrition over the three rounds, which is to be expected with any research; however, the same cohort that commenced round 1 were involved in rounds 2 and 3 . Overall, the participant characteristics highlight that the largest proportion of participants were aged between 40 and 49 years $(31.8 \%, n=7)$, with $22.7 \%(n=5)$ and $18.2 \%(n=4)$ aged between 50 and 59 years and 60-69 years, respectively. There were proportionally more males $(63.6 \%, n=14)$ than females $(31.8 \%, n=7)$, with one (4.5\%) participant reporting other. Naturopathy was the largest professional group represented $(45.5 \%, n=10)$, followed by other $(18.2 \%, n=4)$, western herbalism $(9 \%, n=2)$, traditional Chinese medicine $(9 \%, n=2)$, homeopathy $(4.5 \%, n=1)$, chiropractic $(4.5 \%, n=1)$ and osteopathy $(4.5 \%, n=1)$. Over half $(54.6 \%, n=12)$ of participants had 15 or more years of experience in their field; 32\% $(n=7)$ had 10-14 years of experience, $4.5 \%(n=1)$ had 5-9 years of experience and 9\% $(n=2)$ had $\leq 5$ years of experience in their field. Most respondents $(68.2 \%, n=15)$ held a Doctor of Philosophy, with $13.6 \%$ $(n=3)$ holding a Professional Doctorate, $4.5 \%(n=1)$ holding a Master's degree and $4.5 \%(n=1)$ holding a professional diploma; 9\% $(n=2)$ were Doctor of Philosophy candidates. In terms of geographical location, ten (45.5\%) participants were from Australasia, seven (31.8\%) were from North America, and five (22.7\%) were from Europe.

\section{Interpretation of the Delphi result Delphi - round one}

The first-round survey contained a total of 30 statements that represented the barriers to the conduct and application of research in CAM (Table 3). None of the statements in this round achieved $\geq 50 \%$ for category 0 ("not relevant"), therefore, no statements were removed. Twenty (67\%) statements had reached the $\geq 70 \%$ agreement threshold for the sum of category 2 ("relevant but requires minor revision") and category 3 ("very relevant and requires no revision"), and as such, these statements were included in the final instrument. Nine statements (30\%) did not reach the agreement threshold of $\geq 70 \%$ and thus required revision.

Of the 20 statements added to the final instrument, those examining the barriers to the conduct of CAM 


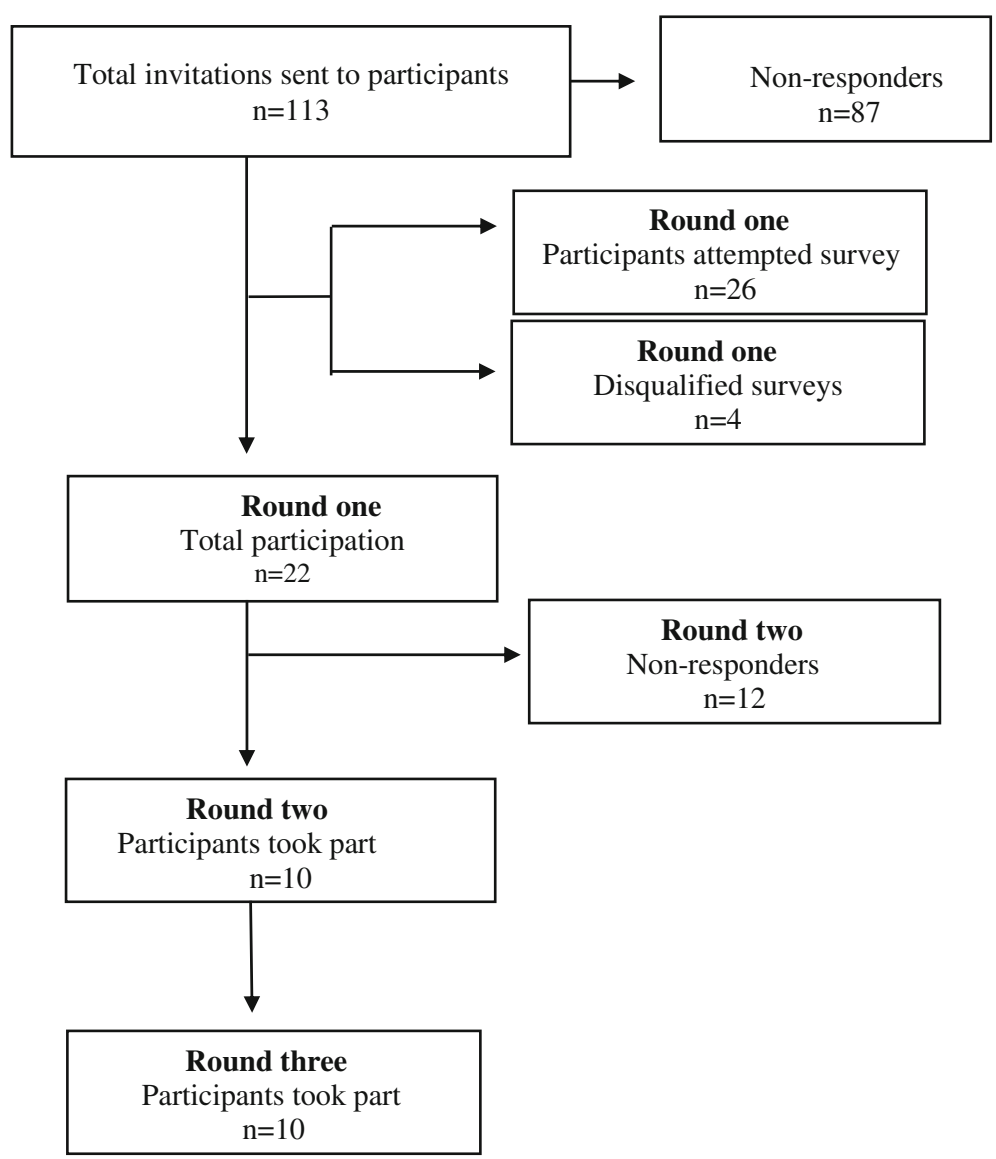

Fig. 2 Flow of participation from round one to round three

research (Part A) included "limited funding" (91\% agreement), "limited opportunities to contribute to research" (82\%), "limited dedicated researchers" (82\%), "limited journal and grant reviewers" (82\%), "negative perceptions of CAM from outside the field of CAM" (82\%), "lack of collaboration between CAM and mainstream researchers" (86\%), "limited dedicated expert CAM researchers" (73\%), "limited recognition for the value of CAM research" (82\%), "limited opportunities for $C A M$ research skill development for undergraduate CAM students" (86\%), and "limited undergraduate opportunities to contribute to research" (86\%).

The statements initially added to the final oBSTACLES instrument that examined the barriers to the application of research in CAM were "limited access to research evidence" (70\% agreement), "limited training" (77\%), "limited awareness of clinical practice guidelines" (77\%), "limited knowledge and skills to locate evidence" (73\%), "appraising research evidence" (94\%), "applying research evidence" (91\%), "inconsistencies within CAM research evidence" (82\%), "limited knowledge and skills to communicate research findings to their patients" (77\%), "little professional obligation to use research evidence to inform their practice" (95\%), "diverse views on what constitutes research evidence" (100\%), and "patient expectations that are contrary to research evidence" (82\%).

All statements that reached $\geq 70 \%$ were accepted and not included in the subsequent Delphi rounds, except in two instances. There were a number of qualitative comments from the panel, which indicated that two statements were confusing and required further clarification; this applied to statements 11 and 23. Statement 11, "There are limited numbers of CAM researchers who have dedicated expertise in a CAM discipline", had received suggestions from participants to change the wording to, "There are a limited number of CAM trained clinician-researchers", and "An adequate number of conventional researchers knowledgeable in CAM". Based on these comments, this statement was split into two individual statements, as follows: "There are a limited number of CAM-trained clinician researchers" and "There are a limited number of mainstream (non-CAM-trained) researchers actively investigating CAM". Similarly, statement 23, "Inconsistencies within CAM research evidence are an obstacle to apply research evidence to practice", received comments from participants 
Table 3 Overview of participant responses to part A and part B items from round one
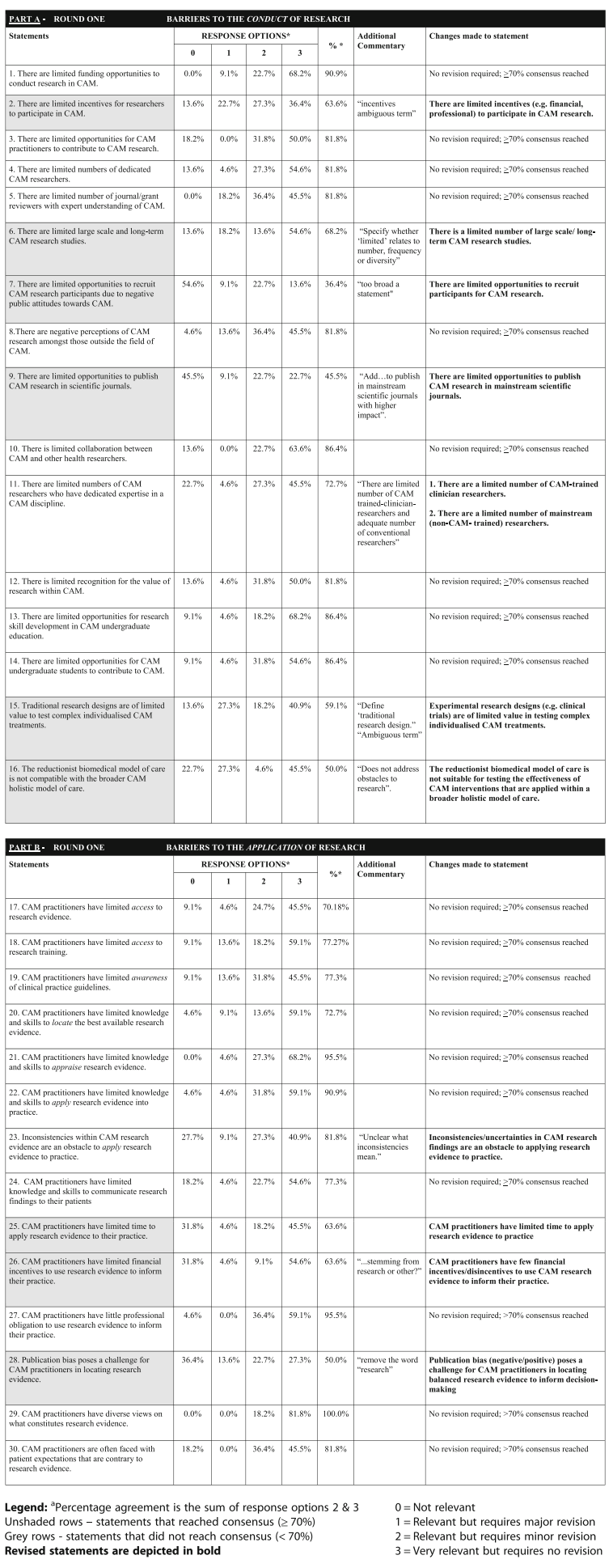

indicating that it was "unclear what inconsistencies mean." Therefore, the statement was changed to, "Inconsistencies/uncertainties in CAM research findings are an obstacle to applying research evidence to practice". These three revised statements were included in the second Delphi round.

Nine statements (30\%) did not reach the agreement threshold of $\geq 70 \%$. These statements related to "limited 
incentives for researchers to participate in CAM" (64\% agreement), "limited large scale and long-term CAM research studies" (68\%), "limited opportunities to recruit CAM research participants due to negative public attitudes towards CAM" (36\%), "limited opportunities to publish CAM research in scientific journals" (45\%), "Traditional research designs are of limited value to test complex individualised CAM treatments" (59\%), "The reductionist biomedical model of care is not compatible with the broader CAM holistic model of care" (50\%), "CAM practitioners have limited time to apply research evidence to their practice" (64\%), "CAM practitioners have limited financial incentives to use research evidence to inform their practice" (64\%), and "publication bias poses a challenge for CAM practitioners in locating research evidence" (50\%). Qualitative comments seeking changes/clarification were reviewed and considered. These nine statements were included in the round two Delphi survey (providing a total of 12 statements to be reviewed).

Table 3 provides an overview of the participant responses from round one. The "clear" rows represent statements that had reached consensus. The "grey" rows represent statements that required further refinement.

\section{Delphi - round two}

The response rate for the second round was $45.5 \%$ $(10 / 22$ round one respondents). None of the statements in the second round achieved $\geq 50 \%$ agreement for category 0 ("not relevant"), and thus no statements were removed from the survey. The second-round analyses determined that 11 out of the $12(92 \%)$ statements had reached the $\geq$ $70 \%$ agreement threshold for the sum of category 2 ("relevant with minor changes") and category 3 ("very relevant and requires no revision"). As such, these statements were included in the final oBSTACLES instrument. One statement (8\%) did not reach the agreement threshold of $\geq 70 \%$ and thus required revision (Table 4).

The six statements from part A of the survey (barriers to the conduct of research in CAM) that achieved $\geq 70 \%$ agreement included "limited incentives to participate in research" (90\% agreement), "limited large-scale/long-term research" (70\%), "limited participant recruitment opportunities" (90\%), "limited CAM trained researchers" (100\%), "experimental research designs are of limited value when testing CAM individualised treatments" (80\%), and "the biomedical model is not suitable for testing CAM interventions" (70\%). With regards to the statement "There is a limited number of large scale/ long-term CAM research studies", while it achieved an agreement rating of $70 \%$, the additional commentary from participants highlighted the following: "Two issues, so have two items, one for large-scale, one for long-term". Therefore, this statement was split into two statements, as follows: "There are limited opportunities to conduct large scale CAM research studies" and "There are limited opportunities to conduct long-term CAM research studies". These two statements were subsequently added to round three.

Statement 6 of part A, "There are a limited number of mainstream (non-CAM trained) researchers", was the only statement in round two that did not achieve a $\geq 70 \%$ agreement rating (reaching only $40 \%$ agreement). Some participants suggested, "It should be worded differently", and it should specify "researchers actively investigating CAM". Therefore, this statement was changed to "There are a limited number of mainstream (non-CAM-trained) researchers actively investigating CAM" and was subsequently added to the round three Delphi survey.

The four statements from part B of the instrument (barriers to the application of research in CAM) that achieved an agreement rating $\geq 70 \%$ included "inconsistent research findings are an obstacle to applying evidence to practice" (80\% agreement), "limited time to apply evidence" (90\%), "few financial incentives to use evidence" (80\%) and "publication bias" (80\%).

A total of three statements (three from part A and zero from part B) were included in the third and final Delphi survey. Table 4 provides an overview of the participant responses from round two.

\section{Delphi - round three}

The response rate for the third round was $100 \%(10 / 10$ round two respondents). All three statements in round three were successful in reaching the $\geq 70 \%$ agreement threshold (Table 5). These statements referred to "limited opportunities to conducting large-scale research" (90\% agreement), "limited opportunities to conduct long-term research" (90\%) and "limited mainstream researchers actively investigating CAM" (70\%). On completion of the third Delphi round, 32 barrier statements that had achieved an agreement rating of $\geq 70 \%$, from all three rounds, were incorporated into the final instrument - the BarrierS To the Application and Conduct of rEsearch (oBSTACLES) (Additional file 1). Table 5 provides an overview of the participant responses from round three.

\section{Discussion}

Despite the increasing focus on CAM to prove its evidence-base, and growing recognition of the numerous barriers to the conduct and application of research within CAM, there has not been a single instrument that has adequately captured and measured these barriers to date. Therefore, the extent to which stakeholders of CAM are impacted by barriers to the conduct AND application of research is largely unknown. While there 
Table 4 Overview of participant responses to part A and part B items from round two

\begin{tabular}{|c|c|c|c|c|c|c|c|}
\hline \multirow[t]{2}{*}{ Statements } & \multicolumn{4}{|c|}{ RESPONSE OPTIONS* } & \multirow{2}{*}{$\%$ * } & \multirow{2}{*}{$\begin{array}{l}\text { Additional } \\
\text { Commentary }\end{array}$} & \multirow[t]{2}{*}{ Changes made to statement } \\
\hline & 0 & 1 & 2 & 3 & & & \\
\hline $\begin{array}{l}\text { 1. There are limited incentives (e.g. } \\
\text { financial. Professional) to participate in } \\
\text { CAM research. }\end{array}$ & $10.0 \%$ & $0.0 \%$ & $30.0 \%$ & $60.0 \%$ & $90.0 \%$ & & No revision required; $>70 \%$ agreement reached \\
\hline $\begin{array}{l}\text { 2. There is a limited number of large } \\
\text { scale/ long-term CAM research studies. }\end{array}$ & $20.0 \%$ & $10.0 \%$ & $10.0 \%$ & $60.0 \%$ & $70.0 \%$ & $\begin{array}{l}\text { "Two issues, so have } \\
\text { two items, one for } \\
\text { large-scale, one for } \\
\text { long-term". }\end{array}$ & $\begin{array}{l}\text { 1. There are limited opportunities to conduct } \\
\text { large scale CAM research studies. } \\
\text { 2. There are limited opportunities to conduct } \\
\text { long-term CAM research studies. }\end{array}$ \\
\hline $\begin{array}{l}\text { 3. There are limited opportunities to } \\
\text { recruit participants for CAM research. }\end{array}$ & $10.0 \%$ & $0.0 \%$ & $30.0 \%$ & $60.0 \%$ & $90.0 \%$ & $\begin{array}{l}\text { "Not relevant to study } \\
\text { conduct". }\end{array}$ & No revision required; $\geq 70 \%$ agreement reached \\
\hline $\begin{array}{l}\text { 4. There are limited opportunities to } \\
\text { publish CAM research in mainstream } \\
\text { scientific journals. }\end{array}$ & $10.0 \%$ & $0.0 \%$ & $10.0 \%$ & $80.0 \%$ & $90.0 \%$ & & No revision required; $\geq 70 \%$ agreement reached \\
\hline $\begin{array}{l}\text { 5. There are a limited number of CAM- } \\
\text { trained clinician researchers. }\end{array}$ & $0.0 \%$ & $0.0 \%$ & $20.0 \%$ & $80.0 \%$ & $100.0 \%$ & & No revision required; $>70 \%$ agreement reached \\
\hline $\begin{array}{l}\text { 6. There are a limited number of } \\
\text { mainstream (non-CAM- trained) } \\
\text { researchers. }\end{array}$ & $40.0 \%$ & $20.0 \%$ & $20.0 \%$ & $20.0 \%$ & $40.0 \%$ & $\begin{array}{l}\text { "It should be worded } \\
\text { differently'; "There } \\
\text { are limited numbers of } \\
\text { mainstream (non- } \\
\text { CAM-trained) } \\
\text { researchers actively } \\
\text { investigating CAM". }\end{array}$ & $\begin{array}{l}\text { There are a limited number of mainstream } \\
\text { (non-CAM-trained) researchers actively } \\
\text { investigating CAM. }\end{array}$ \\
\hline $\begin{array}{l}\text { 7. Experimental research designs (e.g. } \\
\text { clinical trials) are of limited value in. } \\
\text { testing complex individualised CAM } \\
\text { treatments. }\end{array}$ & $0.0 \%$ & $20.0 \%$ & $20.0 \%$ & $60.0 \%$ & $80.0 \%$ & & No revision required; $\geq 70 \%$ agreement reached \\
\hline $\begin{array}{l}\text { 8. The reductionist biomedical model of } \\
\text { care is not suitable for testing the } \\
\text { effectiveness of CAM interventions that } \\
\text { are applied within a broader holistic } \\
\text { model of care. }\end{array}$ & $0.0 \%$ & $30.0 \%$ & $0.0 \%$ & $70.0 \%$ & $70.0 \%$ & & No revision required; $\geq 70 \%$ agreement reached \\
\hline
\end{tabular}

\begin{tabular}{|c|c|c|c|c|c|c|c|}
\hline \multirow[t]{2}{*}{ Statements } & \multicolumn{4}{|c|}{ RESPONSE OPTIONS } & \multirow[b]{2}{*}{$\% *$} & \multirow{2}{*}{$\begin{array}{l}\text { Additional } \\
\text { Commentary }\end{array}$} & \multirow[t]{2}{*}{ Changes made to statement } \\
\hline & $\mathbf{0}$ & 1 & 2 & 3 & & & \\
\hline $\begin{array}{l}\text { 9. Inconsistencies/uncertainties in CAM } \\
\text { research findings are an obstacle to } \\
\text { applying research evidence to practice. }\end{array}$ & $0.0 \%$ & $0.0 \%$ & $0.0 \%$ & $80.0 \%$ & $80.0 \%$ & & No revision required; $>70 \%$ agreement reached \\
\hline $\begin{array}{l}\text { 10. CAM practitioners have limited time } \\
\text { to apply research evidence to practice }\end{array}$ & $10.0 \%$ & $0.0 \%$ & $20.0 \%$ & $70.0 \%$ & $90.0 \%$ & & No revision required; $>70 \%$ agreement reached \\
\hline $\begin{array}{l}\text { 11. CAM practitioners have few financial } \\
\text { incentives/disincentives to use CAM } \\
\text { research evidence to inform their } \\
\text { practice. }\end{array}$ & $10.0 \%$ & $10.0 \%$ & $20.0 \%$ & $60.0 \%$ & $80.0 \%$ & & No revision required; $>70 \%$ agreement reached \\
\hline $\begin{array}{l}\text { 12. Publication bias (negative/positive) } \\
\text { poses a challenge for CAM practitioners } \\
\text { in locating balanced research evidence to } \\
\text { inform decision-making. }\end{array}$ & $0.0 \%$ & $10.0 \%$ & $10.0 \%$ & $80.0 \%$ & $80.0 \%$ & & No revision required; $>70 \%$ agreement reached \\
\hline
\end{tabular}

Legend: $\quad *$ Percentage agreement is the sum of response options $2 \& 3$ Unshaded rows - statements that reached consensus $(\geq 70 \%)$ Grey rows - statements that did not reach consensus $(<70 \%)$ Revised statements are depicted in bold

$0=$ Not relevant

$1=$ Relevant but requires major revision evant but requires minor revision

$3=$ Very rele are generic instruments that explore CAM stakeholder's perspectives of issues such as EBP, these instruments do not capture the range of barriers impacting through the EBP continuum (generation - access - application of research evidence). Therefore, the aim of this research was to refine and validate a dedicated instrument (oBSTACLES instrument) for measuring the barriers to the conduct and application of research in CAM.

Using the Delphi method, the panel of international experts in CAM agreed that myriad barriers exist to the 
Table 5 Overview of participant responses to part A items from round 3

\begin{tabular}{|c|c|c|c|c|c|c|c|}
\hline PART A - ROUND THREE & \multicolumn{6}{|c|}{ BARRIERS TO THE CONDUCT OF RESEARCH } & \\
\hline \multirow[t]{2}{*}{ Statements } & \multicolumn{4}{|c|}{ RESPONSE OPTIONS } & \multirow{2}{*}{$\% *$} & \multirow[t]{2}{*}{ Additional Commentary } & \multirow[t]{2}{*}{ Changes made to statement } \\
\hline & $\mathbf{0}$ & 1 & 2 & 3 & & & \\
\hline $\begin{array}{l}\text { 1. There are limited opportunities to } \\
\text { conduct large scale CAM research } \\
\text { studies. }\end{array}$ & $0.0 \%$ & $10.0 \%$ & $0.0 \%$ & $90.0 \%$ & $90.0 \%$ & & $\begin{array}{l}\text { No revision required } \geq 70 \% \\
\text { agreement reached }\end{array}$ \\
\hline $\begin{array}{l}\text { 2. There are limited opportunities to } \\
\text { conduct long-term CAM research } \\
\text { studies. }\end{array}$ & $0.0 \%$ & $10.0 \%$ & $10.0 \%$ & $80.0 \%$ & $90.0 \%$ & & $\begin{array}{l}\text { No revision required as } \geq 70 \% \\
\text { agreement reached }\end{array}$ \\
\hline $\begin{array}{l}\text { 3. There are a limited number of } \\
\text { mainstream (non-CAM-trained) } \\
\text { researchers actively investigating } \\
\text { CAM. }\end{array}$ & $30.0 \%$ & $0.0 \%$ & $20.0 \%$ & $50.0 \%$ & $70.0 \%$ & & $\begin{array}{l}\text { No revision required as } \geq 70 \% \\
\text { agreement reached }\end{array}$ \\
\hline $\begin{array}{l}\text { *Percentage agreement is } \\
\text { Unshaded rows - stateme } \\
\text { Grey rows - statements th } \\
\text { Revised statements are }\end{array}$ & $\begin{array}{l}\text { sum of } r \\
\text { that reac } \\
\text { did not re } \\
\text { icted in }\end{array}$ & $\begin{array}{l}\text { d consens opt } \\
\text { a consens } \\
\text { ld }\end{array}$ & $\begin{array}{l}2 \& 3 \\
(\geq 70 \%) \\
(<70 \%)\end{array}$ & \multicolumn{3}{|c|}{$\begin{array}{l}0=\text { Not relevant } \\
1=\text { Relevant but requires major revision } \\
2=\text { Relevant but requires minor revision } \\
3=\text { Very relevant but requires no revision }\end{array}$} & \\
\hline
\end{tabular}

conduct and application of research in CAM. The views of the experts are congruent with and supported by contemporary literature [20]. For example, the expert panel concurred that time was an important barrier to research application, and this was consistent with previous reports $[10,12,13,49,50]$. Also supported by previous literature was the view that access to funding was a critical barrier to research conduct [51-56]. Furthermore, the panel of international experts did not reject any statement that had formed the preliminary oBSTACLES instrument (which were developed from the systematic review and NGT). This highlights the consistency of, and consensus amongst, experts on the recognition of myriad barriers encountered when engaging with research.

The resultant output of this research is the oBSTACLES instrument. This self-administered questionnaire comprises 40 items, divided into three parts: Part A (barriers to the conduct of research in CAM; 18 statements), Part B (barriers to the application of research in CAM; 14 statements) and Part C (demographic details; 8 items). All items in parts A and B used a Likert scale response format. The oBSTACLES instrument has familiarity with other survey instruments, such as EBASE and the BARRIERS scale. The BARRIERS [17] scale, for instance, sets out to measure the factors that hinder the application of nursing research, taking into account the researcher, the organisation, and the innovation. EBASE [18], on the other hand, although related to CAM, investigates the factors impacting the clinical application of evidence-based practice, such as practitioner attitude, skills and training. However, where the
oBSTACLES instrument differs from these two instruments is its focus on measuring the barriers to both the conduct and application of research in CAM. This is important, as these barriers may occur throughout the EBP continuum (generation - access - application of research evidence). It is important to capture these barriers throughout the continuum as previous research indicates that access to research alone is not sufficient [57] when promoting EBP; the focus should also be on the application of research [58]. Without such overt focus across the EBP continuum, evidence-practice gaps will continue to persist [59].

The oBSTACLES instrument also acknowledges that effective engagement with CAM research requires a contribution from more than one stakeholder [60]. In fact, the instrument draws attention to the influential role of at least six different stakeholder groups that impact EBP implementation, including researchers, educators, funders, editors/publishers, practitioners and industry (i.e. professional associations). Therefore, the data generated from using the oBSTACLES instrument may shed light on the development of strategies that are more targeted and meaningful to specific stakeholder groups, which in turn, may assist in breaking down barriers to the conduct and application of research in CAM.

As with any research, there are some limitations to this study. First, given the nature of the Delphi study methodology, there were three distinct rounds. This "stop-start-stop" process did not allow for free-flowing exchange of ideas and discussions [61]. However, the use of Delphi study methodology as means of gaining 
consensus on a given topic [62] is well recognised and commonly used in survey development. Second, despite considerable efforts to ensure wide-spread representation across the globe, participants from Asia and Europe were under-represented. Acquiring equal numbers of CAM practitioners and researchers also could not be controlled due to the voluntary nature of the Delphi recruitment process. As this research brought together an international sample of CAM experts from multiple geographical locations, the impact of under-representation was minimised. Third, anonymity due to privacy and confidentiality reasons may have led to a paucity of accountability [63]. Prior to the commencement of the Delphi study, the research team did request all participants to actively and openly contribute to the research. Even though this research provides evidence of consensus on the barriers to the conduct and application of research in CAM, as it used a Delphi study methodology, the findings are considered expert opinion only. Notwithstanding, as this Delphi study included CAM experts from across several other countries, the research arguably captured broad and diverse perspectives and experiences. Furthermore, as the oBSTACLES instrument has its foundation in a systematic review of the literature as well as a nominal group technique, the impact of these limitations has been minimized. Lastly, even though the oBSTACLES instrument may have potential application to areas other than CAM (such as medicine, nursing and allied health), further psychometric testing of the oBSTACLES instrument would be required.

\section{Conclusion}

While there has been widespread recognition of the barriers that confront CAM stakeholders when engaging with research, there has been limited research that has systematically identified and measured what these barriers are across the EBP continuum. This research, by bringing together CAM experts from across the globe, has generated a series of evidence-informed statements, in the form of a survey instrument, to identify, measure and evaluate barriers to the conduct and application of research in CAM. The outcome of this research was the development of the BarrierS to the Application and Conduct of rEsearch (the oBSTACLES instrument) in CAM - a bespoke instrument that can measure and quantify barriers to both the conduct and the application of research in CAM.

\section{Implications for practice}

The oBSTACLES instrument is a simple, easy to use instrument, which is evidence-informed as it has been developed in a methodological manner and underpinned by rigorous research processes (the systematic review, the nominal group technique and the Delphi study). It builds on existing tools such as EBASE and the BARRIERS scale, while addressing their limitations. This instrument can be applied across a range of different practice settings spanning the spectrum of CAM disciplines. As the oBSTACLES instrument measures and quantifies barriers to the conduct and application of research in CAM, it will assist in the development of targeted enabling strategies to overcome these barriers. By doing so, the oBSTACLES instrument provides opportunities to address entrenched barriers that confront CAM stakeholders when engaging with research.

\section{Implications for research}

While the development process for the oBSTACLES instrument was underpinned by a systematic and rigorous methodology, formal psychometric testing remains to be undertaken. Areas of future research include establishing reliability, validity and utility of the oBSTACLES instrument, as well as formal testing with other health professional groups (such as nursing and allied health). With increasing focus on not just the generation of evidence but also on the implementation and evaluation of evidence in health care, the oBSTACLES instrument provides ideal opportunities for future research to examine, measure and understand those barriers that exist not in isolation but across the evidence-based continuum. In turn, the instrument may help to determine what enabling strategies may work best for whom and under what contexts.

\section{Additional file}

Additional file 1: The oBSTACLES instrument. (DOCX $43 \mathrm{~kb}$ )

\section{Abbreviations}

CAM: Complementary and alternative medicine; EBP: Evidence-based practice; OBSTACLES instrument: BarrierS To the Application and Conduct of rEsearch instrument

\section{Acknowledgements}

The authors would like to thank and acknowledge all the participants of this study for generously providing their valuable time, feedback and validation for the development of the oBSTACLES instrument.

\section{Funding}

This study was not supported by any source of funding.

\section{Availability of data and materials}

All data generated or analysed during this study are included in this published article (and its supplementary information files are available from the corresponding author upon request).

\section{Authors' contributions}

SK and ML assisted and guided $\mathrm{Y}$ in refining the Delphi study design, formulating the preliminary OBSTACLES survey instrument, and finalising the oBSTACLES instrument. YV led the participant recruitment with the assistance of SK and ML. YV, ML and SK were all involved in data management and analyses. YV drafted the paper, and ML and SK provided extensive editorial input. All authors reviewed and approved the final manuscript for submission. 


\section{Ethics approval and consent to participate}

Ethics approval was sought from the University of South Australia Human Research Ethics Committee [Protocol number: 000003616]. A Participant Information Sheet detailing the nature of the study and participant's rights were provided in an introductory email. In the first page of the online survey, participants were required to select one of two responses: (a) "I understand and agree to take part" (thus consenting to participate) or (b) "I do not agree to take part" (thus indicating their intent to withdraw from the study).

\section{Consent for publication}

Not applicable.

\section{Competing interests}

The authors declare that they have no competing interests.

\section{Publisher's Note}

Springer Nature remains neutral with regard to jurisdictional claims in published maps and institutional affiliations.

\section{Author details}

'School of Health Sciences, University of South Australia, North Terrace, Adelaide, SA 5000, Australia. ${ }^{2}$ Department of Rural Health, University of South Australia, North Terrace, Adelaide, SA 5000, Australia.

\section{Received: 29 December 2017 Accepted: 16 October 2018} Published online: 14 December 2018

\section{References}

1. World Health Organisation. Who traditional medicine strategy: 2014- 2023. WHO. 2013. http://www.who.int/traditional-complementary-integrativemedicine/en/. Accessed 20 Aug 2018.

2. Rayner J, Willis K, Pirotta M. What's in a name: integrative medicine or simply good medical practice? Fam Pract. 2011. https://doi.org/10.1093/fampra/cmr032.

3. Leach MJ. Clinical decision making in complementary and alternative medicine. 1st ed. Victoria: Elsevier; 2010.

4. Frass $M$, Strassi $R$, Friehs $H$, Mullner $M$, Kundi $M$, Kaye A. Use and Acceptance of complementary and alternative medicine among the general population and medical personnel: a systematic review. Ochsner J. 2012;12(Suppl 1):45-56.

5. Strattoni T, McGivern-Snofsky J. Toward a sociological understanding of complementary and alternative medicine use. J Altern Complement Med. 2007. https://doi.org/10.1089/acm.2007.7006.

6. Brosnan C. Epistemic cultures in complementary medicine: knowledgemaking in university departments of osteopathy and Chinese medicine. Health Sociol Rev. 2016. https://doi.org/10.1080/14461242.2016.1171161.

7. Sarris J. Current challenges in appraising complementary medicine evidence. Med J Aust. 2012. https://doi.org/10.5694/mja11.10751.

8. Ernst E. Obstacles to research in complementary and alternative medicine. Med J Aust. 2003;179(Suppl 6):279-80.

9. Tabish SA. Complementary and Alternative healthcare: is it evidence-based? Int J Health Sci. 2008;2(Suppl 1):5-9.

10. Bussières A, Terhorst L, Leach M, Stuber K, Evans R, Schneider M. Selfreported attitudes, skills and use of evidence-based practice among Canadian doctors of chiropractic: a national survey. J Can Chiropr Assoc. 2015;59(Suppl 4):332-48.

11. Leach MJ, Gillham D. Are complementary medicine practitioners implementing evidence based practice? Complement Ther Med. 2011. https://doi.org/10.1016/j.ctim.2011.04.002.

12. Roecker CB, Lawrence DJ, Long CR, Vining RD. Attitudes toward evidencebased clinical practice among doctors of chiropractic with diplomate-level training in orthopedics. Chiropr Man Therap. 2013. https://doi.org/10.1186/ 2045-709X-21-43.

13. Schneider MJ, Evans R, Haas M, Leach M, Hawk C, Long C. US chiropractors' attitudes, skills and use of evidence-based practice: a cross-sectional national survey. Chiropr Man Therap. 2015. https://doi.org/10.1186/s12998-015-0060-0.

14. Tilburt JC, Curlin FA, Kaptchuk TJ, et al. Alternative medicine research in clinical practice - a US national survey. Arch Intern Med. 2009. https://doi. org/10.1001/archinternmed.2009.49.

15. Evans A: Complementary and alternative medicine (CAM): setting an Australian research agenda. In Proceedings of the 34th annual scientific meeting of the Clinical Oncological Society of Australia (COSA). 2007. http:// www.cosa.org.au/media/1068/cosa_report_complementary-and-alternativemedicine-workshop_2007.pdf. Accessed 16 Oct 2016.

16. Walker BF, Stomski NJ, French SD, Herbert JJ. A survey of Australian chiropractors' attitudes and beliefs about evidence-based practice and their use of research literature and clinical practice guidelines. Chiropr Man Therap. 2013. https://doi.org/10.1186/20145-709X-21-44.

17. Funk SG, Champagne MT, Wiese RA, Tornquist EM. BARRIERS: The barriers to research utilization scale. Appl Nurs Res. 1991. https://doi.org/10.1186/1748-5908-5-32.

18. Leach MJ, Gillham DM. Evaluation of the evidence-based practice attitude and utilization survEy for complementary and alternative medicine practitioners. J Eval Clin Pract. 2008. https://doi.org/10.1111/j.1365-2753.2008.01046.x.

19. Karimian Z, Sabbaghian Z, Saleh A, Sedghpour BS. Obstacles to undertaking research and their effect on research output: a survey of faculty members' views. East Mediterr Health J. 2012;18(Suppl 11):1143-50.

20. Veziari Y, Leach M, Kumar S. Barriers to the conduct and application of research in complementary and alternative medicine: a systematic review. BMC Complement Altern Med. 2017. https://doi.org/10.1186/s12906-017-1660-0.

21. Mcsharry J, Hynes L, Byrne M. Prioritising target behaviours for research in diabetes: using the nominal group technique to achieve consensus from key stakeholders. Res Involv Engagem. 2016. https://doi.org/10.1186/s40900-016-0028-9.

22. Tongco M. Purposive sampling as a tool for informant selection. Ethnobot Res Appl. 2006. https://doi.org/10.17348/era.5.0.147-158.

23. Boulkedid R, Abdoul H, Loustau M, ibony O, Alberti C. Using and reporting the Delphi method for selecting healthcare quality indicators: a systematic review. 2011. https://doi.org/10.1371/journal.pone.0020476.

24. Dalkey N, Hemler O. An experimental application of the Delphi method to the use of experts. Manag Sci. 1963. https://doi.org/10.1287/mnsc.9.3.458.

25. Steurer J. The Delphi method: an efficient procedure to generate knowledge. Skelet Radiol. 2011. https://doi.org/10.1007/s00256-011-1145-z.

26. Mcmillan S, King M, Tully M. How to use the nominal group and Delphi techniques. Int J Clin Pharm. 2016. https://doi.org/10.1007/s11096-016-0257-x.

27. Keeney S, Hasson F, Mckenna H. Consulting the oracle: ten lessons from using the Delphi technique in nursing research. J Adv Nurs. 2006 https://doi.org/10.1111/j.1365-2648.2006.03716.x.

28. Giannarou L, Zervas E. Using Delphi technique to build consensus in practice. Int J Bus Sci Appl Manag. 2014;9:65-82.

29. Skulmoski G, Hartmann F, Krahn J. The Delphi method for graduate research. J Inf Technol Educ. 2007. https://doi.org/10.28945/199.

30. Twycross A, Chorney J, Mcgrath P, Finley G, Boliver D, Mifflin K. A Delphi study to identify indicators of poorly managed pain for paediatric postoperative and procedural pain/Une étude Delphi pour déterminer les indicateurs de douleur mal gérée après Une opération ou pendant Une intervention en pédiatrie. Pain Res Manag. 2013. https://doi.org/10.1155/ 2013/570478.

31. Hsu C, Sandford BA. The Delphi technique: making sense of consensus. Pract Assess. 2007;12(Suppl 10):1-8.

32. Hasson F, Keeney S, Mckenna H. Research guidelines for the Delphi survey technique. J Adv Nurs. 2000. https:/doi.org/10.1046/j.1365-2648.2000.t01-1-01567x. .

33. Powell C. The Delphi technique: myths and realities. J Adv Nurs. 2003. https://doi.org/10.1046/j.1365-2648.2003.02537.x.

34. Slade SC, Dionne CE, Underwood M, Buchbinder R. Standardised method for reporting exercise programmes: protocol for a modified Delphi study. BMJ Open. 2014. https://doi.org/10.1136/bmjopen-2014-006682.

35. Geisser ME, Alschuler KN, Hutchinson R. A Delphi study to establish important aspects of ethics review. J Empir Res Hum Res Ethics. 2011. https://doi.org/10.1525/jer.2011.6.1.21

36. Ludwig B. Predicting the future: have you considered using the Delphi methodology? J Ext. 1997;35:1-4.

37. Helmy R, Zullig LL, Dunbar-Jacob J, Hughes DA, Vrijens B, Wilson IB. ESPACOMP medication adherence reporting guidelines (EMERGE): a reactive-Delphi study protocol. BMJ Open. 2017;7. https://doi.org/10.1136/ bmjopen-2016-013496

38. Sedgwick P. Snowball sampling. BMJ. 2013. https://doi.org/10.1136/bmj.f7511.

39. Noy C. Sampling knowledge: the hermeneutics of snowball sampling in qualitative research. Int J Soc Res Methodol. 2008. https://doi.org/10.1080/ 13645570701401305

40. Dillman LM, Smyth J, Christian LM. Internet, Phone, mail, and mixed-mode surveys: the tailored design method. 4th ed. Canada: Wiley; 2014.

41. Sinha IP, Smyth RL, Williamson PR. Using the Delphi technique to determine which outcomes to measure in clinical trials: recommendations for the 
future based on a systematic review of existing studies. PLoS Med. 2011. https://doi.org/10.1371/journal.pmed.1000393.

42. Keeney S, Mckenna H, Hasson F. The Delphi technique in nursing and Health Research. 1st ed. Oxford: Wiley-Blackwell; 2011.

43. Waclawski E. How I use it: survey monkey. Occup Med. 2012. https://doi.org/ 10.1093/occmed/kas075.

44. Banwell HA, Mackintosh S, Thewlis D, Landorf KB. Consensus-based recommendations of Australian podiatrists for the prescription of foot orthoses for symptomatic flexible pes planus in adults. J Foot Ankle Res. 2014. https://doi.org/10.1186/s13047-014-0049-2.

45. Kleynen M, Braun SM, Bleijlevens MH, Lexis MA, Rasquin SM, Halfens J, et al. Using a Delphi technique to seek consensus regarding definitions, descriptions and classification of terms related to implicit and explicit forms of motor learning. PLoS One. 2014. https://doi.org/10.1371/journal.pone.0100227.

46. Hicks C. Research methods for clinical therapists: applied project design and analysis. 5th ed. Edinburgh: Churchill Livingstone; 2009.

47. Vernon W, Parry A, Potter M. Consensus obtained in a Delphi study of shoe wear pattern experiences amongst podiatrists. J Forensic Identif. 2003;53(Suppl 1):15-41.

48. Day J, Bobeva M. A Generic toolkit for the successful Management of Delphi Studies. Electron J Bus Res Methodol. 2005.

49. Alcantara J, Leach M. Chiropractic attitudes and utilization of evidencebased practice: the use of the EBASE questionnaire. Explore. 2015. https://doi.org/10.1016/j.explore.

50. Scherer RW, Ugarte-Gil C, Schmucker C, Meerpohl JJ. Authors report lack of time as main reason for unpublished research presented at biomedical conferences: a systematic review. J Clin Epidemiol. 2015. https://doi.org/10. 1016/j.jclinepi.2015.01.027.

51. Bensoussan A, Lewith GT. Complementary medicine research in Australia: a strategy for the future. Med J Aust. 2004;181(Suppl 6):331-3.

52. Ernst E. Funding research into complementary medicine: the situation in Britain. Complement Ther Med. 1999. https://doi.org/10.1016/S0965-2299(99)80011-9.

53. Grace S. CAM practitioners in the Australian health workforce: an underutilized resource. BMC Complement Altern Med. 2012. https://doi.org/ 10.1186/1472-6882-12-205

54. Giordano J, Engebretson J, Garcia MK. Challenges to complementary and alternative medical research: focal issues influencing integration into a Cancer care model. Integr Cancer Ther. 2005. https://doi.org/10.1177/1534735405279179.

55. Lewith G, Holgate S. CAM research and development. Complement Ther Nurs Midwifery. 2000. https://doi.org/10.1054/ctnm.1999.0440.

56. Wardle J, Adams J. Are the CAM professions engaging in high-level health and medical research? Trends in publicly funded complementary medicine research grants in Australia. Complement Ther Med. 2013. https://doi.org/10 1016/j.ctim.2013.09.003.

57. Hanson D, Allegrante JP, Sleet DA, Finch CF. Research alone is not sufficient to prevent sports injury. Br J Sports Med. 2014. https://doi.org/10.1136/ bjsports-2012-091434.

58. Jackson CL, Janamian T, Van WC, Dunbar JA. Implementation research - its importance and application in primary care: Moving evidence from theoretical to practical levels requires a collaborative effort. Med J Aust. 2014. https://doi.org/10.5694/mja14.00063.

59. Carrington SJ, Uljarević M, Roberts A, et al. Knowledge acquisition and research evidence in autism: researcher and practitioner perspectives and engagement. Res Dev Disabil. 2016. https://doi.org/10.1016/j.ridd.2016.01.011.

60. Aarons GA, Wells RS, Zagursky K, Fettes DL, Palinkas LA. 2009 implementing evidence-based practice in community mental health agencies: a multiple stakeholder analysis. Am J Public Health. 2009. https://doi.org/10.2105/AJPH. 2009.161711.

61. Courtney M, O'reilly M, Edwards H, Hassall S. Content validity of the ResCareQA: an Australian residential care quality assessment based on resident outcomes. Aust J Adv Nurs. 2011;28(Suppl 3):37-46.

62. Dars $\mathrm{S}$, Uden $\mathrm{H}$, Kumar $\mathrm{S}$, Banwell HA. When, why and how foot orthoses (FOs) should be prescribed for children with flexible pes planus: a Delphi survey of podiatrists. Peer J. 2018. https://doi.org/10.7717/peerj.4667.

63. Sackman H. Delphi critique; expert opinion, forecasting, and group process. Massachusetts: Lexington books; 1975.

\section{Ready to submit your research? Choose BMC and benefit from:}

- fast, convenient online submission

- thorough peer review by experienced researchers in your field

- rapid publication on acceptance

- support for research data, including large and complex data types

- gold Open Access which fosters wider collaboration and increased citations

- maximum visibility for your research: over $100 \mathrm{M}$ website views per year

At BMC, research is always in progress.

Learn more biomedcentral.com/submissions 